\title{
Electronic nicotine delivery systems (ECs) and COVID-19: the perfect storm for young consumers
}

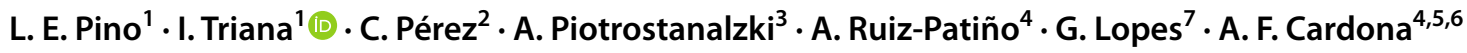

Received: 6 May 2020 / Accepted: 10 May 2020 / Published online: 23 May 2020

(c) Federación de Sociedades Españolas de Oncología (FESEO) 2020

\begin{abstract}
The COVID-19 pandemic caused a change in our society and put health systems in crisis worldwide. Different risk factors and comorbidities have been found that increase the risk of mortality when acquiring this infection. The use of alternative devices to the cigarette like the electronic cigarettes, the vapers have been studied widely and generators of great controversy since it has been discovered that they also produce different pulmonary affections. When developing the SARS-CoV2 infection, different theories have been generated about the greater predisposition to a worse prognosis of people who use electronic cigarettes; however, the information on this continues in discovery. A group of experts made up of oncologists, infectologists, pulmonologists, and epidemiologists met to review the literature and then generate theories about the impact of electronic cigarettes on SARS-CoV2 infection.
\end{abstract}

Keywords Electronic cigarettes $\cdot$ Macrophages $\cdot$ SARS-CoV2 $\cdot$ COVID-19 $\cdot$ Viral infection $\cdot$ Pulmonary infection

\section{Introduction}

COVID-19 is a coronavirus outbreak that initially appeared in Wuhan, China, but it has already evolved into a pandemic spreading rapidly worldwide [1]. As of 4 April 2020, a total number of 1,201,591 cases of COVID-19 have been reported, including 64,703 deaths, the majority of which have been reported in China Italy (15.362), Spain (11.947), and France (7.560) [2]. Information from China, where COVID-19 originated, shows that people who have cardiovascular and respiratory conditions caused by tobacco use,

\section{Triana}

Camilotrianaa@gmail.com

L. E. Pino

Docpino2@gmail.com

A. Piotrostanalzki

audreypvh@gmail.com

A. Ruiz-Patiño

Alejandro.ruiz.pat@gmail.com

G. Lopes

Glopes.med@gmail.com

A. F. Cardona

a_cardonaz@yahoo.com

1 Cancer Institute Fundación Santafé, 110121 Bogotá,

Colombia or otherwise, are at higher risk of developing severe symptoms [3]. Research on 55,924 laboratory-confirmed cases in China shows that the crude fatality ratio for COVID-19 patients is much higher among those with cardiovascular disease, diabetes, hypertension, chronic respiratory disease or cancer than those with no pre-existing chronic medical conditions [4]. This demonstrates that these pre-existing conditions may contribute to increasing the susceptibility of such individuals to COVID-19.

Smoking is also detrimental to the immune system and its responsiveness to bacterial and viral pathogens, making

2 Infectious Disease Department Clínica, Marly - Hospital La Samaritana, 110121 Bogotá, Colombia

3 Respiratory Medicine Department, Clínica Central del Quindío, 110121 Armenia, Colombia

4 Clinical and Traslational Oncology Group, Institute of Oncology, Country Clinic, 110121 Bogotá, Colombia

5 Foundation for Clinical and Applied Cancer Research - FICMAC, 110121 Bogotá, Colombia

6 Molecular Oncology and Biology Systems Research Group (Fox-G), Universidad El Bosque, 110121 Bogotá, Colombia

7 Sylvester Comprehensive Cancer Center, Miami University, Miami, USA 
smokers more vulnerable to infectious diseases [5]. Previous studies have shown that smokers are twice more likely than non-smokers to contract influenza and have more severe symptoms, while smokers were also noted to have higher mortality in the previous MERS-CoV outbreak [6, 7]. Recently, Zhang et al. [8] presented clinical characteristics of 140 patients with COVID-19. The results showed that among severe patients $(n=58), 3.4 \%$ were current smokers and $6.9 \%$ were former smokers, in contrast to non-severe patients $(n=82)$ among which $0 \%$ were current smokers and $3.7 \%$ were former smokers, leading to an OR for an adverse event of 2.23; (95\% CI 0.65-7.63; $p=0.2$ ). In addition, Guan et al. reported in an epidemiological analysis that those who either needed mechanical ventilation, admission to an ICU or died, $25.5 \%$ were current smokers and $7.6 \%$ were former smokers. In contrast, in the group of patients that did not have these adverse outcomes, only $11.8 \%$ were current smokers and $1.6 \%$ were former smokers (no statistical analysis for evaluating the association between the severity of the disease outcome and smoking status was conducted in that study) [9]. From this limited information, it has been possible to calculate that the smokers were 1.4 times more likely (RR 1.4, 95\% CI 0.98-2.00) to have severe symptoms of COVID-19 and approximately 2.4 times more likely to be admitted to an ICU, need mechanical ventilation or die compared to non-smokers (RR 2.4, 95\% CI 1.43-4.04) [10].

In the US, approximately $3.7 \%$ of adults use electronic cigarettes (EC), and 1.8 million adolescents also vape, and this number is rising. Lung-induced injury (VpALI) is a well-recognized entity. So far, now we know that COVID19 also affect younger people; in a cohort of Chinese Center for Disease Control and Prevention comprised of 44,500 cases, $87 \%$ of patients were between 30 and 79 years old [11]. In another Chinese cohort, only $2.6 \%$ of the patients were under 20 years old. Out of 9241 cases diagnosed in South Korea, approximately $6.3 \%$ were in the same range of age ( $\approx 580$ cases), [12] distribution that in Italy reaches a case-fatality rate of $0.7 \%$ ( 575 patients aged between 30 and 50 years old). In the study for China cited above, there was a mortality of $0.6 \%$ for individuals between the ages of 10 and 40 years (190 cases) [13]. While mortality is relatively low for young patients, deaths still occur, and EC use may be a potential risk factor similar to what happens with tobacco. Considering the mortality estimates for the youngest and the risk index for smokers, it is possible that for the estimated 93,530 deaths from COVID-19 expected in the US for August 2020 (https://covid19.healthdata.org/), about $\approx 1400$ will be young EC consumers.

Molecular analysis has begun to shed light on how SARS-CoV-2 infections occur. Like a related coronavirus that emerged in 2003, SARS-CoV-2 enters human cells by binding to the extracellular domain of Angiotensin Converting Enzyme 2 (ACE2) [14, 15]. Importantly, ACE2 is both necessary and sufficient for infection by SARS-CoV-2: ACE2-targeting antibodies block viral uptake in permissive cells while transgenic expression of human ACE2 allows viral entry in non-human cells. ACE2 normally functions in the renin-angiotensin system (RAS) by cleaving the vasoconstrictive hormone angiotensin-II into the vasodilator angiotensin [16]. Sequestration of ACE2 by coronavirus dysregulates the RAS pathway, contributing to morbidity. In addition, ACE2 levels are capable of influencing disease progression [17].

Recently, Smith et al. found that smoker's lung expresses 40-50\% more ACE2 compared to tissue from non-smokers, being the samples with the highest receptor expression, those belonging to smokers who reported the highest exposure index in number of pack-years [17]. For instance, among smokers undergoing thoracic surgery, patients who had smoked more than 80 pack-years exhibited $\mathrm{a} \sim 100 \%$ increase in ACE2 expression relative to patients who had smoked less than 20 pack-years $[17,18]$. Furthermore, multivariate linear regression analysis on this dataset further confirmed that smoking history was a significant predictor of ACE2 expression even when controlling for a patient's age, sex, race, and body-mass index [17].

Otherwise, contemporary analysis has been geared towards the constituents of E-liquids/E-juice or vaping products like Vitamin-E (alpha-tocopherol) acetate (VEA) which is being implicated as the likely 'exogenous' source of lipids in these ECs-user subjects [19, 20], and perhaps a causal factor, because it was detected in the bronchoalveolar lavage fluids (BALF) of several cases with e-cigarette or vaping product use associated lung injury (EVALI) [21, 22]. For e-liquids, VEA (retinoic acid) is used as an additive to dissolve/dilute THC oils along with mineral, and triglyceride medium chain oil, and is also used as a thickening agent for other non-THC e-liquids. Thus far, in a nationwide US study, VEA, natural oil (coconut derivate), and terpene have been identified in $94 \%, 2 \%$, and $3 \%$ of EVALI patient BALF samples, respectively [23]. The absence of these compounds in healthy comparators makes VEA the potential causative agent for EVALI. VEA or its oxidant/radical derivatives is harmful when inhaled/vaporized interfering with phospholipids and surfactant of the epithelial lining fluid, event that triggers a severe lipoid pneumonitis [24].

In a chronic format, exposure to inhaled nicotine and other irritants generated by ECs disrupts RAS homeostasis through inhibition of local compensatory mechanisms, leading to increased susceptibility to cardiovascular and pulmonary distress, hypothetically increasing the risk generated by COVID-19, similar to the way it does in tobacco smokers. By other hand, among EC users, a significant increase in MUC4, a membrane-anchored mucin, and an increase in the ratio of secretory mucins MUC5AC to MUC5B has been reported compared to non-users [21]. A cross-sectional 
study suggested inflammasome complex proteins, caspase-1 and apoptosis-associated speck-like protein containing caspase activation and recruitment domain (ASC), which promote cellular pyroptosis, are elevated in the BALF of EC consumers [25]. In deep biological single cell analysis demonstrated that ACE2 expression increased among smokers in both MUC5AC goblet cells and SCGB1A1 club cells, increasing the probability of SARS-CoV-2 adhesion and favoring its alveolar damage [17].

In patients with EVALI, there was a higher concentration of serum C-reactive protein (CRP) [26], finding that is reproduced in patients with respiratory distress syndrome (ARDS) related to severe COVID-19. Recently, Luo et al. described the usefulness of CRP in COVID-19, finding that this marker exhibits a sensitivity of $90.5 \%$, a specificity of $77.6 \%$, a positive predictive value of $61.3 \%$, and a negative predictive value $95.4 \%$. In the same study, a subgroup analysis revealed that CRP retained a robust accuracy to predict adverse outcome in patients with different disease severity (AUC 0.832, $p<0.001$ and AUC 0.989, $p<0.001$ ) and represent an independent discriminator of severe/critical illness on admission (AUC 0.783, $p<0.001$ ) [27].

Some studies have reported increased inflammatory cell influx in the lung of patients with EVALI, including significantly altered expression of early growth response (EGR1), ZBTB16, PIGR, PTGS2, and FKBP5 compared to the occasional EC users with reduced CSF-1, CCL26, and eotaxin-3 levels that are essential for the mucosal host-defense [28,
29]. In another study, Lerner et al. demonstrated increased BALF IL-6 and CCL2 levels following EC aerosol exposure [30], one of the pillars of SARS-CoV-2-induced alveolar damage. Furthermore, the binding of SARS-CoV-2 to the Toll-Like Receptor (TLR) causes the release of pro-IL-1 $\beta$ which is cleaved by caspase-1, followed by inflammasome activation and production of active mature IL- $1 \beta$ which is a mediator of lung inflammation, fever and fibrosis. Suppression of pro-inflammatory IL-1 family members and IL-6 have been shown to have a therapeutic effect in many inflammatory diseases, including severe viral infections. Several compounds has the ability to suppress innate and acquired immune response and also has the capacity to inhibit inflammation by acting on IL1 and 6, IL17, and 18. This cytokines inhibits class II histocompatibility complex (MHC) molecules and inflammation in inflammatory diseases by suppressing MyD88 and subsequently IL-1 $\beta$, IL-6, TNF and CCL2 [31].

Pod-based e-juices and flavors also induce cellular toxicity with identification of several toxic chemicals [32], hence this will render users susceptible to further damage as seen in EVALI cases. Similarly, Wang et al. reported dysregulated lung repair following EC exposure due to higher levels of Angiopoietin-1 and CXCL5 with lower MMP3 levels, indicating the involvement of the tissue-remodeling pathways $[33,34]$. Other rodent model studies have shown potential of DNA damage, adduct formation, and genotoxicity/carcinogenicity of EC and vaping product aerosols, additive

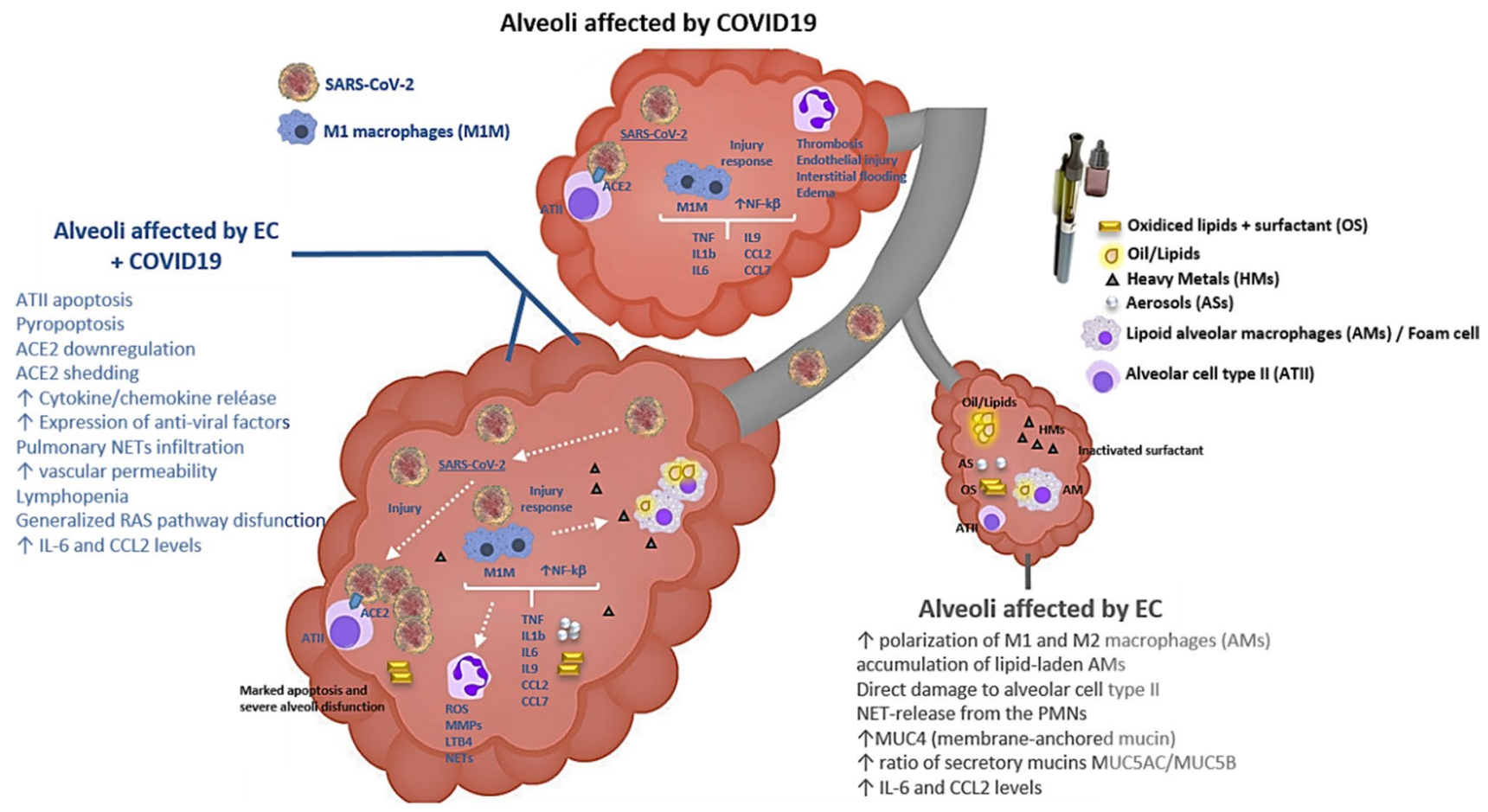

Fig. 1 The double hit effect 
findings to the fibrosis damage generated by coronaviruses. Figure 1 generates an explicative hypothesis about the "double hit effect" generated by exposure to ECs plus SARSCoV-2 infection in the lung.

The factors that mediate susceptibility to SARS-CoV-2 infections are poorly understood. We speculate that the increased expression of ACE2 in the lungs of smokers and EC consumers could partially contribute to the severe cases of COVID-19 that have been observed. Despite the fact that COVID-19 disease generates higher mortality in older adults, people younger than 50 years exposed to toxic inhalants such as EC should be considered as patients with pulmonary immunosuppression and, therefore, seen as being at higher risk of infection severity. There is currently insufficient evidence to confirm this relationship, but different opinions and trends have already been reported around the world regarding the increased risk of mortality in young people with active EC when infected with COVID-19. Just a week ago, the CDC (Center for Disease Control, Atlanta, US) reported that young adults under age 44 make up a big part of COVID-19 hospitalizations in the US, and health providers questions whether the vaping epidemic might have contributed to this [35].

We strongly recommend that than doctors linked to tobacco cessation programs and the World Health Organization (WHO) rapidly advance their efforts to reduce smoking and EC consumption, especially during COVID-19 pandemic. It is important that The Framework Convention on Tobacco Control (FCTC) Secretariat issue expeditious and useful recommendations that allow public health decisionmaking for the protection of young people suffering from SARS-CoV-2 infection while actively using ECs. Similarly, lines of research about this topic must be prioritized by public health agencies.

\section{Contributorship}

No additional contribution was received.

Funding For the development of this article, no external funds were received from any institution or person. The costs were covered by the authors of the study.

\section{Compliance with ethical standard}

Conflict of interests No author or co-author has a conflict of interest in the publication or writing of this article.

Ethics statement This is a risk-free study, without intervention with patients. With the follow-up of all relevant ethical considerations. Attached to the statement of Declaration of Helsinki of the Association World Medical in its version of the year 2013.

\section{References}

1. Wu JT, Leung K, Leung GM. Nowcasting and forecasting the potential domestic and international spread of the 2019-nCoV outbreak originating in Wuhan, China: a modelling study. Lancet. 2020;395(10225):689-97. https://doi.org/10.1016/S0140 $-6736(20) 30260-9$.

2. Dong E, Du H, Gardner L. An interactive web-based dashboard to track COVID-19 in real time. Lancet Infect Dis; published online. https://doi.org/10.1016/S1473-3099(20)30120-1. (link: https://gisanddata.maps.arcgis.com/apps/opsdashboard/index .html\#/bda7594740fd40299423467b48e9ecf6) (Accessed March $162020)$.

3. World Health Organization. Report of the WHO-China Joint Mission on Coronavirus Disease 2019 (COVID-19), 14-20 2020. (https://www.who.int/docs/default-source/coronaviruse/whochina-joint-mission-on-covid-19-final-report.pdf) (Accessed March 16 2020).

4. Guan WJ, Liang WH, Zhao Y, et al. China medical treatment expert group for Covid-19. Comorbidity and its impact on 1590 patients with Covid-19 in China: A Nationwide Analysis. Eur Respir J. 2020;10(1183/13993003):00547-2020.

5. Zhou Z, Chen P, Peng H. Are healthy smokers really healthy? Tob Induc Dis. 2016. https://doi.org/10.1186/s12971-016-0101-z.

6. Park JE, Jung S, Kim A. MERS transmission and risk factors: a systematic review. BMC Public Health. 2018;18(1):574. https:// doi.org/10.1186/s12889-018-5484-8.

7. Arcavi L, Benowitz NL. Cigarette smoking and infection. Arch Intern Med. 2004;164(20):2206-16. https://doi.org/10.1001/archi nte.164.20.2206.

8. Zhang JJ, Dong X, Cao YY, et al. Clinical characteristics of 140 patients infected by SARS-CoV-2 in Wuhan China. Allergy. 2020. https://doi.org/10.1111/all.14238.

9. Guan WJ, Ni ZY, Hu Y, et al. China medical treatment expert group for Covid-19. Clinical characteristics of coronavirus disease in China. N Engl J Med. 2020. https://doi.org/10.1056/NEJMo a2002032.

10. Vardavas CI, Nikitara K. COVID-19 and smoking: A systematic review of the evidence. Tob Induc Dis. 2020;18:20.

11. Wu Z, McGoogan JM. Characteristics of and important lessons from the coronavirus disease 2019 (COVID-19) outbreak in China: Summary of a Report of 72,314 cases from the Chinese center for disease control and prevention. JAMA 2020.

12. KCDC. Updates on COVID-19 in Korea. March 14, 2020. https ://www.cdc.go.kr/board/board.es?mid=a30402000000\&bid=0030 (Accessed March 14 2020).

13. Onder G, Rezza G, Brusaferro S. Case-Fatality Rate and Characteristics of Patients Dying in Relation to COVID-19 in Italy. JAMA Published online March 232020.

14. Li W, et al. Angiotensin-converting enzyme 2 is a functional receptor for the SARS coronavirus. Nature. 2003;426:450-4.

15. Zhou $P$, et al. A pneumonia outbreak associated with a new coronavirus of probable bat origin. Nature. 2020;579:270-3.

16. Hoffmann M, et al. SARS-CoV-2 Cell entry depends on ACE2 and TMPRSS2 and is blocked by a clinically proven protease inhibitor. Cell. 2020. https://doi.org/10.1016/j.cell.2020.02.052.

17. Smith J, Sheltzer J. Cigarette smoke triggers the expansion of a subpopulation of respiratory epithelial cells that express the SARS-CoV-2 receptor ACE2. bioRxiv 2020.03.28.013672; https ://doi.org/10.1101/2020.03.28.013672 (https://www.biorxiv.org/ content/10.1101/2020.03.28.013672v1.full.pdf)

18. Steiling $\mathrm{K}$, et al. A dynamic bronchial airway gene expression signature of chronic obstructive pulmonary disease and lung function impairment. Am J Respir Crit Care Med. 2013;187:933-42. 
19. Sommerfeld CG, Weiner DJ, Nowalk A, et al. Hypersensitivity pneumonitis and acute respiratory distress syndrome from E-cigarette use. Pediatrics. 2018;141(6):e20163927. https://doi. org/10.1542/peds.2016-3927.

20. Viswam D, Trotter S, Burge PS, et al. Respiratory failure caused by lipoid pneumonia from vaping e-cigarettes. BMJ Case Rep. 2018. https://doi.org/10.1136/bcr-2018-224350.

21. Reidel B, Radicioni G, Clapp PW, et al. E-Cigarette use causes a unique innate immune response in the lung, involving increased neutrophilic activation and altered mucin secretion. Am J Respir Crit Care Med. 2018;197:492-501. https://doi.org/10.1164/ rccm.201708-15900C.

22. Moritz ED, Zapata LB, Lekiachvili A, et al. Update: characteristics of patients in a national outbreak of E-cigarette, or vaping, product use-associated lung injuries - United States. MMWR Morb Mortal Wkly Rep. 2019;6-8:985-9. https://doi. org/10.15585/mmwr.mm6843e1.

23. Blount BC, Karwowski MP, Shields PG, et al. (Vitamin E acetate in bronchoalveolar-lavage fluid associated with EVALI. N Engl J Med. 2019. https://doi.org/10.1056/NEJMoa1916433.

24. Xue Y, Williams TL, Li T, et al. Type II pneumocytes modulate surfactant production in response to cigarette smoke constituents: restoration by vitamins A and E. Toxicol Vitro. 2019;19:1061-9. https://doi.org/10.1016/j.tiv.2005.05.003.

25. Tsai M, Song MA, Mcandrew C, et al. Electronic versus combustible cigarette effects on inflammasome component release into human lung. Am J Respir Crit Care Med. 2019;199:922-5. https ://doi.org/10.1164/rccm.201808-1467LE.

26. Kalininskiy A, Bach CT, Nacca NE, et al. E-cigarette, or vaping, product use associated lung injury (EVALI): case series and diagnostic approach. Lancet Respir Med. 2019;7(12):1017-26. https ://doi.org/10.1016/S2213-2600(19)30415-1.

27. Luo X, Zhou W, Yan X, et al. Prognostic value of C-reactive protein in patients with COVID-19. medRxiv 2020.03.21.20040360. https://doi.org/10.1101/2020.03.21.20040360 (link https://www. medrxiv.org/content/10.1101/2020.03.21.20040360v1)

28. Layden JE, Ghinai I, Pray I, et al. Pulmonary illness related to E-cigarette use in Illinois and Wisconsin - preliminary report. $\mathrm{N}$ Engl J Med. 2019. https://doi.org/10.1056/NEJMoa1911614.
29. Martin EM, Clapp PW, Rebuli ME, et al. E-cigarette use results in suppression of immune and inflammatory-response genes in nasal epithelial cells similar to cigarette smoke. Am J Physiol Lung Cell Mol Physiol. 2016;311:L135-L144144. https://doi.org/10.1152/ ajplung.00170.2016.

30. Lerner CA, Sundar IK, Yao H, et al. Vapors produced by electronic cigarettes and E-juices with flavorings induce toxicity, oxidative stress, and inflammatory response in lung epithelial cells and in mouse lung. PLoS ONE. 2015;10:e0116732. https://doi. org/10.1371/journal.pone.0116732.

31. Conti P, Ronconi R, Caraffa A. Induction of pro-inflammatory cytokines (IL-1 and IL-1) and lung inflammation by COVID-19: anti-inflammatory strategies. J Biol Regul Homeostas Agents 2020 34(2) 10.23812/CONTI-E

32. Muthumalage T, Lamb T, Friedman MR, et al. E-cigarette flavored pods induce inflammation, epithelial barrier dysfunction, and DNA damage in lung epithelial cells and monocytes. Sci Rep. 2019;9:19035. https://doi.org/10.1038/s41598-019-51643-6.

33. Wang S, Sun NN, Zhang J, et al. Immunomodulatory effects of high-dose alpha-tocopherol acetate on mice subjected to sidestream cigarette smoke. Toxicology. 2002;175:235-45.

34. Crotty LE, Drummond CA, Hepokoski M, et al. Chronic inhalation of e-cigarette vapor containing nicotine disrupts airway barrier function and induces systemic inflammation and multiorgan fibrosis in mice. Am J Physiol Regul Integr Comp Physiol. 2018;314:R834 R847847. https://doi.org/10.1152/ajpregu.00270.2017.

35. CNN Health. How smoking, vaping and drug use might increase risks from Covid-19. March 2020. Available at: https://editi on.cnn.com/2020/03/20/health/coronavirus-vaping-drugs/index .html. (Accessed on March 23 2020).

Publisher's Note Springer Nature remains neutral with regard to jurisdictional claims in published maps and institutional affiliations. 